\title{
Case Report: Extremely Premature Infant without Spontaneous Movements Born to a Mother following COVID-19 Infection
}

Shaina Khan Lodhi, MD

\section{Delivery Course:}

The neonatal intensive care unit (NICU) team was called to the delivery of an infant at 24 weeks of gestation. The mother had been transferred from a referring hospital with complaints of decreased fetal movements. There was a non-reassuring fetal heart rate due to absent variability and a low biophysical profile (BPP) of $2 / 8$ on the first examination at the birth hospital, so the decision was made to proceed with an emergent $\mathrm{C}$-section.

\section{"At the time of delivery, NICU was present in an anteroom next to the operating room due to isolation for COVID-19. The infant was limp and non- vigorous, with a heart rate of $<100$, and positive pressure ventilation (PPV) was started immediately on arrival to the warmer."}

Of note, the mother had tested positive for COVID-19 two weeks prior to the delivery.

\section{Neonatal Resuscitation:}

At the time of delivery, NICU was present in an anteroom next to the operating room due to isolation for COVID-19. The infant was limp and non-vigorous, with a heart rate of $<100$, and positive pressure ventilation (PPV) was started immediately on arrival to the warmer. The infant was noted to have a previously undiagnosed cleft lip and palate on the left, which made ventilation difficult. Despite adequate PPV, the heart rate did not improve. Endotracheal intubation was challenging, and the infant was successfully intubated at 14 minutes of life. The heart rate increased immediately following intubation; however, the infant's saturations remained low, $20-30 \%$ while on $100 \%$ oxygen with PPV. The infant was transferred to an isolation room in the NICU, where he was placed on a high-frequency jet ventilator, with improvement in the saturation and the color of the infant. However, the infant's tone remained extremely poor, and no spontaneous movements or breathing effort was noted.

\section{Readers can also follow} NEONATOLOGY

via our Twitter Feed

@NEO
Apgar scores were:

1 (0 color, 0 reflex, 0 resp, 0 tone, $1 \mathrm{HR}$ ) at 1 minute.

1 (0 color, 0 reflex, 0 resp, 0 tone, $1 \mathrm{HR}$ ) at 5 minute.

1 (0 color, 0 reflex, 0 resp, 0 tone, $1 \mathrm{HR}$ ) at 10 minute.

3 (0 color, 0 reflex, 1 resp, 0 tone, $2 \mathrm{HR}$ ) at 15 minute.

4 (0 color, 0 reflex, 2 resp, 0 tone, $2 \mathrm{HR}$ ) at 20 minute.

Assessment:

Birth weight:

$\begin{array}{ll}\text { Birth weight: } & 955 \mathrm{~g} \\ \text { Birth length: } & 32.5 \mathrm{~cm}\end{array}$

This male infant was large for gestational age and, on the exam, appeared very edematous. Modified Ballard examination was consistent with a gestational age of 24 weeks. Unilateral left cleft lip and palate was noted, without any other evident anomalies. The infant was profoundly hypotonic with no spontaneous movements, and no reflexes were elicited.

\section{Review of Maternal Records:}

On further review of maternal records and discussion with the mother, it was revealed that the mother had symptoms of an upper respiratory infection 2.5 weeks prior to delivery and then had a worsening cough and shortness of breath. She was hospitalized for two days but did not require ventilator support. She tested positive for COVID-19 during this hospitalization. Due to her hospitalization and COVID-19 positive status, she was unable to go for her scheduled fetal anatomy scan. Besides the positive COVID-19 test, prenatal tests were unremarkable.

She had noticed a lack of spontaneous fetal movements three days prior to the delivery. She had gone to the referring hospital the same day, and although the initial BPP was reassuring, all the subsequent ones were low, at $2 / 8$, scored for amniotic fluid index. Delivery was offered but declined initially. The mother received a 2-day course of steroids, after which delivery was offered again and accepted. However, the parents requested to be transferred to a center better equipped to care for extremely premature infants, which had prompted a transfer to the birth hospital.

\section{Hospital Course:}

In the NICU, the infant was started on total parenteral nutrition and continued on the high-frequency jet ventilator. Due to persistently low arterial blood pressures, a dopamine drip was initiated and titrated. Antibiotics were initiated after obtaining a blood culture. A head ultrasound was done on the first day of life, given the prolonged resuscitation and lack of spontaneous activity in the infant, and showed slightly prominent ventricles without evidence of intracranial hemorrhage. Cerebral and renal near-infrared spectroscopy (NIRS) was started per protocol.

Due to the findings of cleft lip and palate, echocardiography and abdominal ultrasounds were ordered to look for other congenital anomalies. The echocardiogram was normal, and abdominal ultrasound demonstrated some free fluid adjacent to the liver. A chromosomal microarray was sent. 
At 20 hours of age, the infant continued to have profound hypotonia and a lack of any spontaneous movements or respiratory effort. The mother of the infant was updated in person regarding these findings. The infant was transitioned from the high-frequency ventilator to a conventional ventilator on the assist control/volume guarantee mode. Amplitude integrated electroencephalography (aEEG) was initiated. SARS-COV2 RNA test done from a nasopharyngeal swab for the infant at 24 hours of life was negative.

At 42 hours of age, there was no improvement in the neurological exam. There continued to be no spontaneous movement or respiratory effort, and the infant remained severely hypotonic. He was not reactive to exam or painful stimuli. Both pupils were dilated and fixed. Cerebral NIRS was mostly above $85 \%$, and aEEG demonstrated a burst suppression pattern with a severely suppressed background. Given these findings and prenatal history, the parents decided to transition to comfort care. The infant was extubated in the mother's arms and passed away soon after, at 47 hours of age.

\section{Post-mortem results:}

An autopsy was performed and was significant for an infant between 24-26 weeks' gestation with a unilateral cleft lip and palate, moderate facial hirsutism, small body cavity effusions, heavy lungs with congestion, edema, and patchy mild airspace hemorrhage, and mild congestive hepatosplenomegaly.

Placental pathology demonstrated moderate to marked decidual arteriopathy with fibrinoid necrosis, low placental weight for gestational age (<10th percentile), parenchyma with accelerated villous maturation for gestational age, and trivascular umbilical cord and membranes with no significant inflammation. The combination of decidual arteriopathy, low placental weight, and accelerated villous maturation supported the diagnosis of maternal vascular malperfusion.

The chromosomal microarray detected several large regions of homozygosity (3 megabases or larger), encompassing nearly $2 \%$ of the genome. Although this result was not diagnostic of a specific genetic condition, it raises the possibility of an autosomal recessive disorder due to the presence of a homozygous mutation in one of these regions.

\section{Discussion:}

The 2014 executive summary of a joint workshop including the American Academy of Pediatrics and American College of Obstetricians and Gynecologists defines periviability as 20 0/7 through 25 6/7 weeks of gestation and recommends antenatal corticosteroids, Cesarean delivery for fetal indications, and aggressive newborn resuscitation for an infant at $230 / 7$ weeks or more unless considered nonviable based on individual circumstances (1). This infant was considered periviable at the time of delivery and underwent aggressive resuscitation. However, upon further review of the maternal records, the infant had had no spontaneous movements noticeable by the mother or on a biophysical profile for several days prior to the delivery, which arguably, may have been an indication to limit the extent of resuscitative efforts offered. This infant likely had an event that neurologically devastated him prenatally and led to a loss of movement. After delivery, the neurological status remained poor, and chances of recovery were low, which led to transitioning to comfort care in this infant.

The cause of this infant's lack of movements at 24 weeks of gestation is unclear. The presence of the cleft lip and palate and a large region of homozygosity found on the chromosomal microarray, although not fatal on their own, could be indicative of an autosomal recessive disorder due to the presence of a homozygous mutation (2). Additionally, the placental pathology showed a small placenta for gestational age and decidual arteriopathy consistent with maternal vascular malperfusion. Maternal vascular malperfusion is found in less than $1 \%$ of uncomplicated pregnancies but at higher frequencies in patients with preeclampsia, fetal death, small for gestational age neonates, and spontaneous preterm labor. Additionally, maternal vascular malperfusion lesions may be associated with abnormal uterine artery flow and placental infarction, affecting neonatal outcomes (3). Therefore, poor oxygen delivery to the fetal brain due to a compromised placenta may have led to the loss of spontaneous movements in this case.

\section{"More recently, it has been shown that placentas from mothers who tested positive for COVID-19 have an increased prevalence of decidual arteriopathy and other features of maternal vascular malperfusion as compared to historical controls (4)."}

More recently, it has been shown that placentas from mothers who tested positive for COVID-19 have an increased prevalence of decidual arteriopathy and other features of maternal vascular malperfusion as compared to historical controls (4). In a review article, 69 of 150 placentas following maternal SARS-CoV-2 infection showed features of maternal vascular malformation (5). However, these are preliminary studies, and at this time, the data is insufficient to conclude causality. This remains an area of interest, and further studies are needed to determine whether maternal COVID-19 infection can be implicated as a cause of maternal vascular malperfusion and subsequent poor neonatal outcomes.

\section{References:}

1. Raju TNK, Mercer BM, Burchfield DJ, Joseph GF. Periviable Birth: Executive Summary of a Joint Workshop by the Eunice Kennedy Shriver National Institute of Child Health and Human Development, Society for Maternal-Fetal Medicine, American Academy of Pediatrics, and American College of Obstetricians and Gynecologists. Obstetrics \& Gynecology. 2014 May;123(5):1083-96.

2. Sund KL, Zimmerman SL, Thomas C, Mitchell AL, Prada CE, Grote L, et al. Regions of homozygosity identified by SNP microarray analysis aid in the diagnosis of autosomal recessive disease and incidentally detect parental blood relationships. Genet Med. 2013 Jan;15(1):70-8.

3. Ernst LM. Maternal vascular malperfusion of the placental bed. APMIS. $2018 \mathrm{Jul} ; 126(7): 551-60$.

4. Shanes ED, Mithal LB, Otero S, Azad HA, Miller ES, Goldstein JA. Placental Pathology in COVID-19. American Journal of Clinical Pathology. 2020 Jun 8;154(1):23-32.

5. Sharps MC, Hayes DJL, Lee S, Zou Z, Brady CA, Almoghrabi $Y$, et al. A structured review of placental morphology and histopathological lesions associated with SARS-CoV-2 infection. Placenta. 2020 Nov;101:13-29.

The author has no relevant disclosures. Verbal consent for this case report was obtained from mother. 


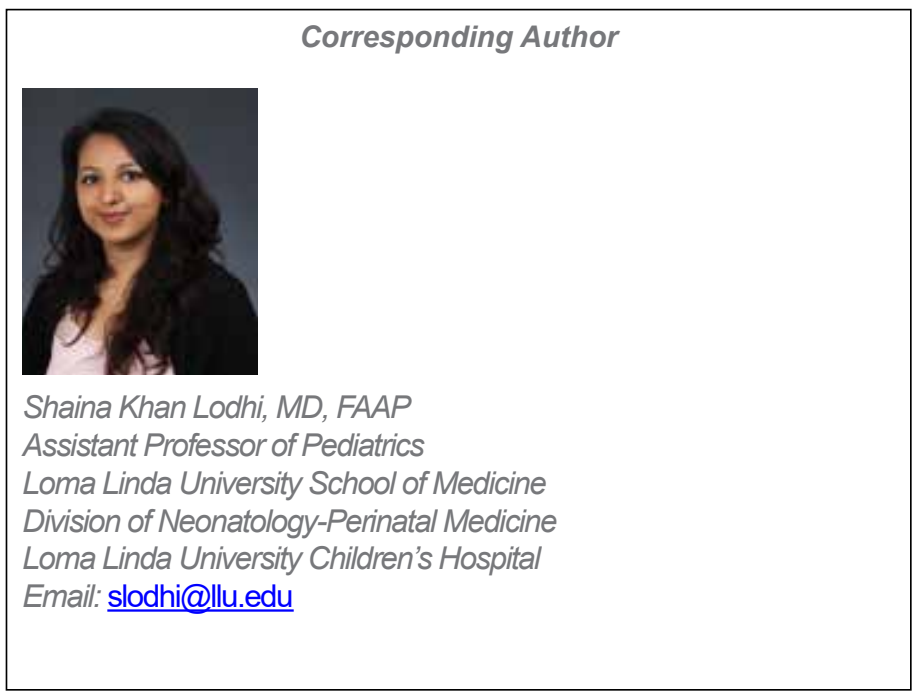

\section{EACH YEAR}

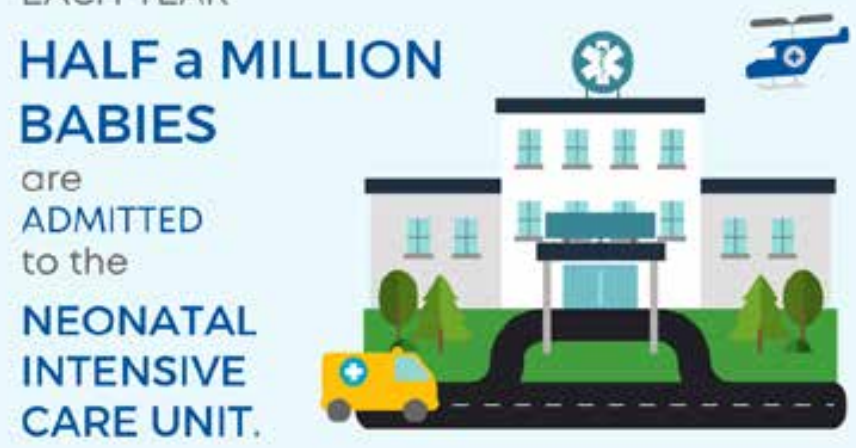

Learn how to support these families.
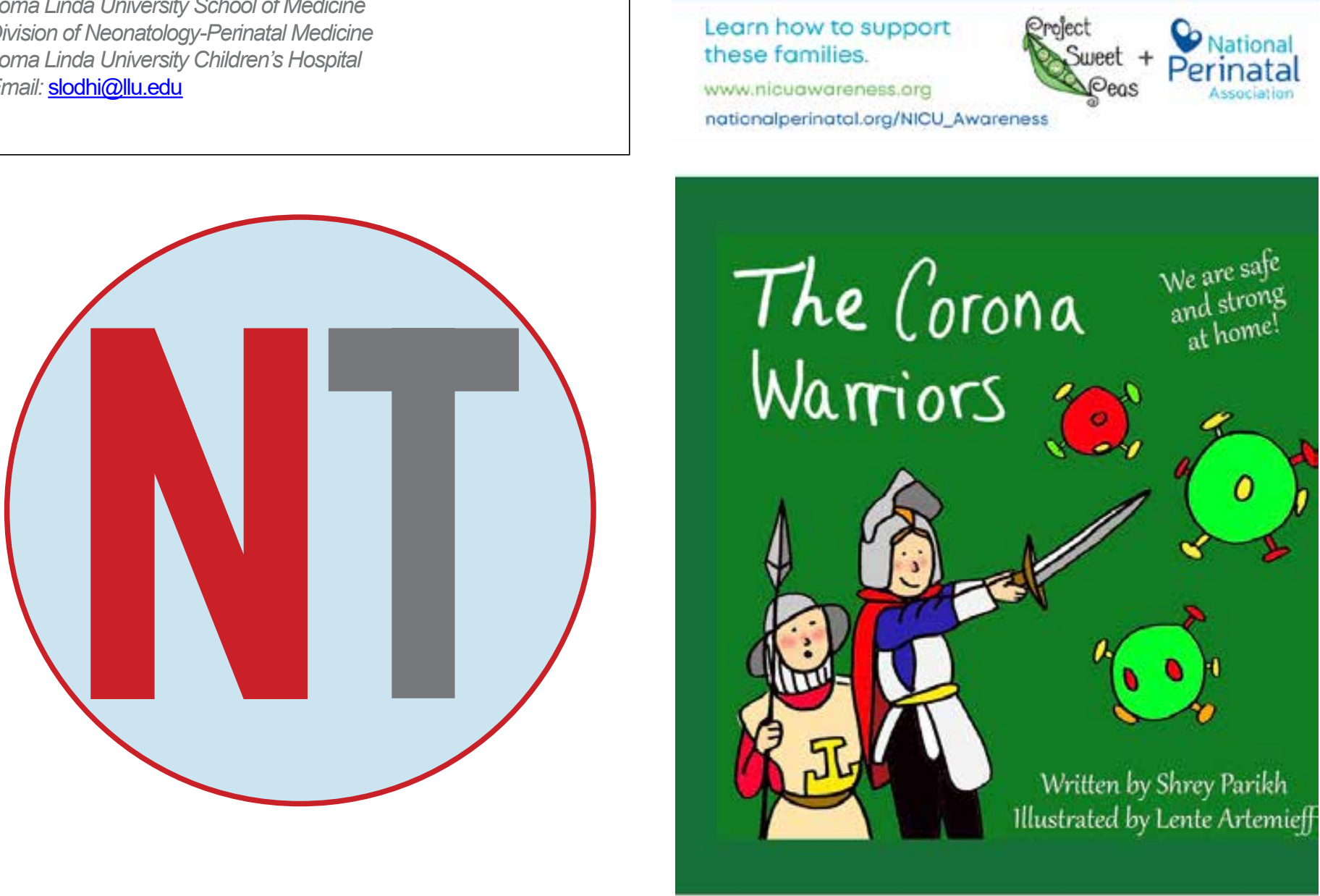

\section{THE \\ BRETT TASHMAN

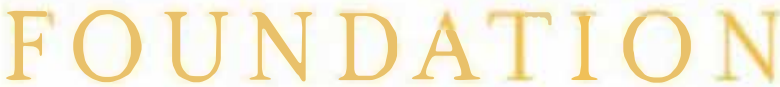

The Brett Tashman Foundation is a 501()(3) public charity. The mission of the Foundation is to find a cure for Desmoplastic Small Cell Round Tumors (DSRCT). DSRCT is an aggressive pediatric cancer for which there is no cure and no standard treatment. 100 percent of your gift will be used for research. There is no paid staff. To make your gift or for more information, go to "TheBrettTashmanFoundation.org" or phone (909) 981-1530. 